\title{
Avoiding Routine Chest Radiography after Transbronchial Biopsy Is Safe
}

\author{
Gabriel Izbicki Ayal Romem Nissim Arish Clement Cahan Hava Azulai \\ Chen Chen-Shuali Esther Tennenhaus Zipi Bar-Yosef Evgeniya Zlotkevich
}

Ariel Rokach

Pulmonary Institute, Shaare Zedek Medical Center, Jerusalem, Israel

\section{Key Words}

Bronchoscopy · Pneumothorax - Chest radiography .

Transbronchial biopsy

\begin{abstract}
Background: Fiberoptic bronchoscopy (FOB) with transbronchial biopsy (TBB) is complicated by a pneumothorax in $1-4 \%$ of cases. Performance of routine post-TBB chest radiography (CXR) results in an extremely low diagnostic yield but nevertheless is the common clinical practice prevailing today. It has previously been suggested that routine post-TBB CXR could be avoided in asymptomatic patients. Objective: The objective of this study was to prospectively assess the feasibility and safety of this approach. Methods: The study group included 201 consecutive patients who underwent FOB with TBB at our institution between January 2009 and September 2014. All subjects completed a preprocedural, a 2-hour postprocedural, and a 24- to 48hour postprocedural symptom questionnaire (chest pain, dyspnea, and cough). Post-TBB CXR was ordered by the treating physician only if indicated. All cases of pneumothorax were documented. Additionally, the following information was recorded: sex, age, immune status, indication for FOB, total number of biopsies done, lobe sampled, and
\end{abstract}

pulse oxygen saturation. Results: Sixteen CXRs were ordered by the treating physician due to suspected pneumothorax (8\%). Early-onset pneumothorax (i.e. within $2 \mathrm{~h}$ of TBB) was diagnosed radiologically in 6 patients (3\%). Two late-onset pneumothoraxes (1\%) were diagnosed more than $24 \mathrm{~h}$ after TBB. No pneumothoraxes of clinical significance were diagnosed among asymptomatic patients without significant oxygen desaturation events. Conclusions: Among asymptomatic patients without significant desaturation events, pneumothorax is rare and usually of negligible clinical significance. Therefore, performance of routine CXR after TBB is not necessary and can be safely avoided in this category of patients. $02016 \mathrm{~S}$. Karger AG, Basel

\section{Introduction}

Since its introduction in the 1960s, fiberoptic bronchoscopy (FOB) has become a defining diagnostic as well as therapeutic procedure for pulmonologists [1].

Trial Registry: ClinicalTrials.gov No. NCT00544479; www.clinicaltrials. gov.

\section{KARGER}

E-Mail karger@karger.com

www.karger.com/res (c) 2016 S. Karger AG, Basel

0025-7931/16/0923-0176\$39.50/0
Ayal Romem, MD, MHA

Pulmonary Institute, Shaare Zedek Medical Center

PO Box 3235

Jerusalem 91031 (Israel)

E-Mail pulmoromem@szmc.org.il 
Though considered a safe procedure with low morbidity and exceptionally low mortality rates, FOB with transbronchial biopsy (TBB) is complicated by pneumothorax in $1-4 \%$ of the cases [2-6]. This has led most pulmonologists and some of the professional societies to endorse routine acquisition of chest radiography (CXR) after TBB, despite a lack of randomized clinical trials to support this practice [7-9]. Indeed, routine postprocedural CXR incurs added costs, time, and patient exposure to radiation [10]. Therefore, the clinical necessity of this approach has been previously challenged [11]. In a prior publication in Chest [4], we have shown that the likelihood of clinically significant pneumothorax among patients lacking any symptoms suggestive of pneumothorax after TBB is low enough to preclude the need for routine CXR. We proposed that routine CXR following bronchoscopy with TBB be obtained only in patients with symptoms suggestive of pneumothorax. In the present study, we prospectively applied our proposed clinical approach with the aim of assessing its feasibility and safety.

\section{Materials and Methods}

All consecutive patients referred for FOB with fluoroscopically guided TBB at the Shaare Zedek Medical Center, Jerusalem, Israel, between January 2009 and September 2014 were approached regarding study enrollment. Only those able and willing to sign an informed consent were recruited. Moderate sedation during FOB was achieved with a combination of intravenous midazolam, fentanyl, and propofol (2,6-diisopropylphenol) as deemed appropriate by the endoscopist. Prior to the procedure, each patient was questioned regarding existing symptoms including chest pain, dyspnea, and cough ( 0 = no symptom; 1 = mild symptoms; 2 = moderate symptoms, and 3 = severe symptoms). The patient was asked to answer the same questionnaire $2 \mathrm{~h}$ after the procedure and again between 24 and $48 \mathrm{~h}$ after the procedure (telephone interview). Vital signs (pulse oximetry, heart rate, and blood pressure) were monitored during the endoscopy and throughout the 2-hour recovery period. Postprocedure CXR films were obtained only in case the endoscopist or another treating physician approached by the patient (emergency room physician, primary care physician) had a high clinical suspicion for pneumothorax or in case of significant bleeding (i.e. the need for cold saline and/or adrenaline instillation intrabronchially). The CXR films were examined by radiologists blinded to the study protocol and to the patient's clinical status. The presence (or absence) of pneumothorax was recorded, in addition to the clinical management (hospitalization, chest tube) if necessary. Further information collected included: sex and age, immune status, indication for the procedure, number of biopsies performed, segment from which most of the biopsy specimens were obtained, and biopsy forceps size. The study was approved by the Shaare Zedek institutional review board (approval No. 72-07).

Avoiding Routine Post-TBB CXR Is Safe

\section{Statistical Analysis}

Simple logistic regression analysis was applied to calculate the odds ratio $(\operatorname{Exp}(B))$ and its significance ( $\mathrm{p}$ values between the variables). The significance of differences in the distribution of the categorical variables between patients with or without pneumothorax was analyzed by $\chi^{2}$ test or Fisher's exact test, as appropriate. Student's t test was used to evaluate differences in continuous variables between two groups. Predictors of pneumothorax were evaluated using logistic regression analysis based on a sample of 199 patients of whom 8 had pneumothorax. A stepwise logistic regression model was fitted to the data in order to predict the presence of pneumothorax after bronchoscopy. Independent variables included in the model were: postprocedural change in cough, chest pain and dyspnea score as well as the occurrence of a persistent desaturation event ( $>5 \%$ drop lasting $\geq 30$ s) during the 2 -hour recovery period; $\mathrm{p} \leq 0.05$ was considered statistically significant.

\section{Results}

Out of 291 consecutive patients undergoing TBB in our institute between January 2009 and September 2014, $201(69 \%)$ were recruited to the study. All other patients (31\%) were either unable to sign the informed consent or declined to do so. The patients' average age was $58.8 \pm 14$ years and roughly half were male $(\mathrm{n}=108)$. Indications for FOB with TBB were: suspected cancer $(n=62,30.8 \%)$; persistent infiltrates $(\mathrm{n}=34,16.9 \%)$; interstitial lung disease $(\mathrm{n}=13,6.5 \%)$; suspected sarcoidosis $(\mathrm{n}=48,23.9 \%)$, and others $(\mathrm{n}=44,21.9 \%)$. Seven patients $(3.5 \%)$ were considered immunosuppressed at the time of the procedure. Midazolam, fentanyl, and propofol were administered to 98,79 , and $24 \%$ of the patients, respectively. Small biopsy forceps (FB-15C, diameter $2 \mathrm{~mm}$; Olympus Optical, Tokyo, Japan) were used in $29 \%$ of the bronchoscopies, and large forceps (FB-20C, diameter $2.8 \mathrm{~mm}$; Olympus Optical) were used in $71 \%$. The distribution of biopsy specimens obtained per procedure was: $1-3$ in $23 \%$; $4-5$ in $52 \%$; $6-7$ in $20 \%$, and $>7$ in $5 \%$. Biopsy specimens were taken from the right upper lobe in $21 \%$ of patients, from the right middle lobe in $17 \%$, from the right lower lobe in $36 \%$, from the left upper lobe in $18 \%$, and from the left lower lobe in $9 \%$ of the patients. Among $18 \%$ of the patients, a second different lobe was also biopsied. All the procedures were done or supervised by a senior pulmonologist.

Pneumothorax was diagnosed radiographically in 8 of the 201 patients ( $4 \%$ of all TBB procedures). The characteristics of the 8 patients are presented in table 1. Six of the pneumothorax cases ( $3 \%$ of all TBB procedures) were clinically suspected by the endoscopist, either immediately or within the 2-hour recovery period, prompting the 
Table 1. Characteristics of patients with radiologically proven pneumothorax following bronchoscopy with $\mathrm{TBB}^{\mathrm{a}}$

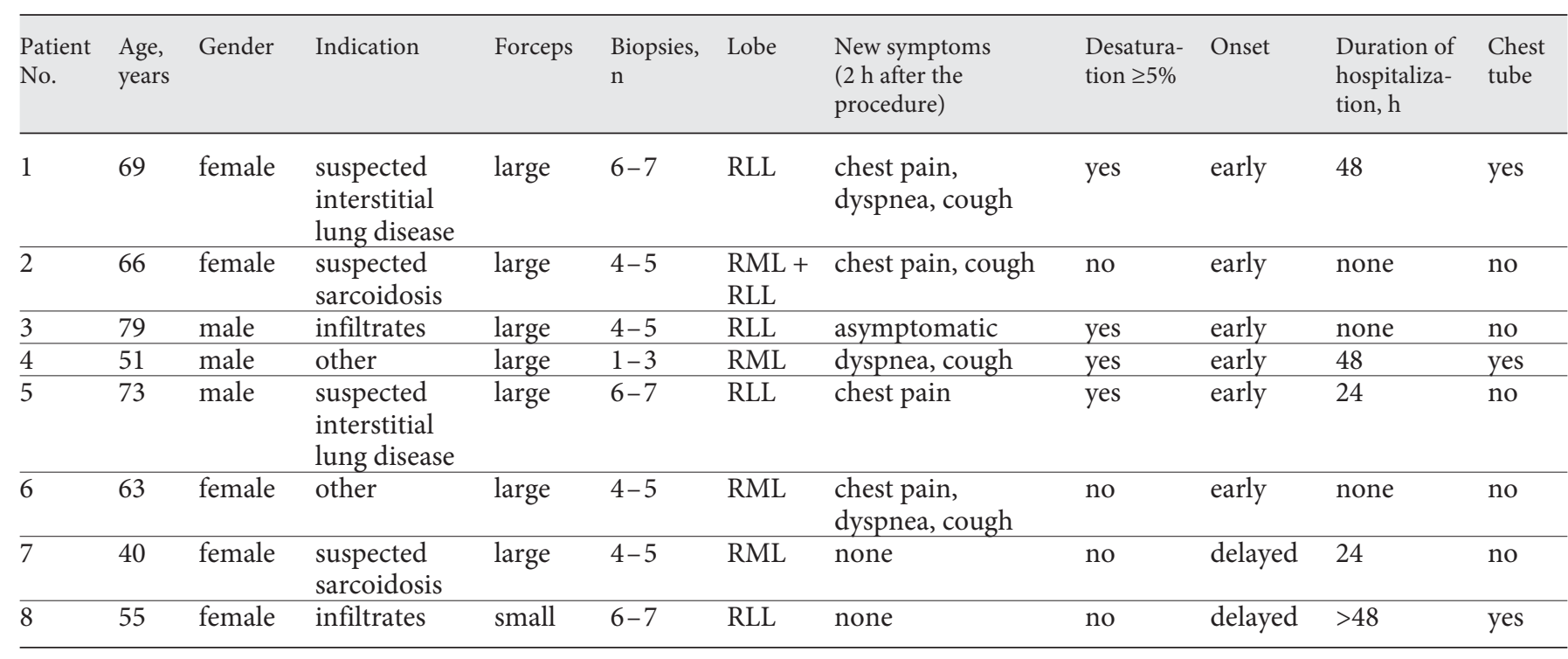

$\mathrm{RLL}=$ Right lower lobe; $\mathrm{RML}=$ right middle lobe. ${ }^{\mathrm{a}}$ The large forceps was $2.8 \mathrm{~mm}$ in diameter, the small forceps $2.0 \mathrm{~mm}$ in diameter.

clinician to order a confirmatory CXR. In 2 of the 6 cases ( $1 \%$ of all the TBB procedures), placement of a chest tube was required. The 2 other pneumothorax cases were of late onset, the first presenting to the emergency department $26 \mathrm{~h}$ after the procedure complaining of new-onset chest pain and dyspnea and subsequently admitted for a 24-hour observation period without the need for chest tube placement. The second case was incidentally discovered 9 days later when the patient came in for a planned surgical procedure. In neither of the 2 cases did the patient report any changes in symptoms during the 2-hour recovery time frame.

During the 2-hour postprocedure observation period, 18 CXRs were ordered by the performing physician, 2 out of concern for a parenchymal bleed and the remaining 16 due to suspected pneumothorax. Among these cases, a CXR was confirmatory in 6 , which is a detection rate of $37.5 \%$. When taking into consideration the size of forceps used, the number of biopsies taken, or the biopsy site, none of these parameters was significantly associated with occurrence of pneumothorax. Positive predictors of pneumothorax included new onset or worsening of chest pain, shortness of breath, and/or cough $2 \mathrm{~h}$ after the procedure. The occurrence of a persistent desaturation event during the 2-hour recovery period was also strongly correlated with the development of a pneumothorax (see table 2 for details). When the data were fitted to a multivariate logistic regression model, the new onset or aggra-
Table 2. Two-hour postprocedural parameters associated with pneumothorax occurrence

\begin{tabular}{lclr}
\hline Symptom/sign & $\begin{array}{l}\text { Odds } \\
\text { ratio }\end{array}$ & $\begin{array}{l}\text { 95\% confidence } \\
\text { interval }\end{array}$ & p value \\
\hline Worsening dyspnea & 22.51 & $4.19-121.59$ & $<0.001$ \\
Worsening chest pain & 18.3 & $3.98-84.1$ & $<0.001$ \\
Worsening cough & 4.4 & $1.02-19.07$ & 0.048 \\
Desaturation & 19.0 & $4.19-121.59$ & $<0.001$ \\
\hline
\end{tabular}

Logistic regression analysis.

vation of chest pain and desaturation remained the only statistically significant parameters associated with pneumothorax (Nagelkerke's $\mathrm{R}^{2}$ of 0.494 and an $\operatorname{EXP}(\mathrm{B})$ value of 198.36 for postprocedural desaturation and 18.98 for chest pain at $2 \mathrm{~h}$ ).

\section{Discussion}

To the best of our knowledge, the present study is the first prospective study to assess the clinical feasibility and safety profile of avoiding routine CXR performance following FOB with TBB. In the current study, the vast majority of patients (96\%) had no evidence of either early or delayed radiographic pneumothorax. Overall, 16 CXRs 
were ordered (only $8 \%$ of all TB done) within $2 \mathrm{~h}$ of procedure completion due to high clinical suspicion (desaturation, worsening chest pain, cough, or dyspnea) of the clinician performing the bronchoscopy. Of those, 6 were found by CXR to have early-onset pneumothorax (i.e. within $2 \mathrm{~h}$ after the procedure). Thus, we were able to avoid routine chest X-ray in $92 \%$ of all patients undergoing FOB with $\mathrm{TBB}$ and to increase the diagnostic yield of the CXR to $37.5 \%$.

Only 2 patients who were asymptomatic during the immediate postprocedure period were subsequently found to have late-onset pneumothorax, the first becoming symptomatic $26 \mathrm{~h}$ after the procedure and managed conservatively without a need for chest tube insertion. The other was incidentally found, more than 1 week after FOB, to have a pneumothorax after obtaining a CXR before undergoing a prescheduled surgical procedure under general anesthesia and positive pressure ventilation, the latter mandating placement of a chest tube despite the fact that the patient was asymptomatic. The 2 patients found by CXR to have early-onset pneumothorax requiring treatment were both symptomatic, and physicians suspected pneumothorax before CXR in both of them. Since only symptomatic patients had CXR, the incidence of subclinical pneumothorax in this series cannot be ascertained. Nevertheless, the prospective nature of the study which included a minimal follow-up of $48 \mathrm{~h}$ practically rules out any clinically significant late-onset pneumothorax. These findings are in accordance with prior retrospective studies showing a very low diagnostic yield with routine post-TBB chest roentgenograms. In their retrospective study, Milam et al. [10] reviewed 207 FOB procedures of which only $47 \%$ included TBB. A total of 130 CXRs (67\%) were obtained with only 1 pneumothorax found in a patient already suspected of having a pneumothorax based on new-onset symptoms and fluoroscopy findings. The authors concluded that postbronchoscopic CXR rarely adds any additional clinically useful information.

Frazier et al. [11], in a retrospective study of 305 routine CXRs following TBB, found no pneumothoraxes in patients who did not have both chest pain and the appearance of lung collapse on pre-CXR fluoroscopy. They concluded that the absence of both symptoms simultaneously effectively rules out pneumothorax. However, both studies mentioned above were conducted retrospectively with all of the inherent limitations attributed to this methodology.

In a previous study, we [4] prospectively recruited 350 patients undergoing TBB procedures and recorded both patient-related as well as procedure-related data while performing routine postprocedural CXRs in all patients. In this study, pneumothorax was detected in 10 patients (2.9\%), out of which 7 had symptoms highly suggestive of pneumothorax prior to CXR. The other 3 patients were asymptomatic with small-size pneumothorax which resolved completely without any need for intervention. Thus, we concluded that routine CXR in asymptomatic patients is not necessary after TBB. Of note, more than a quarter of the study population were post-lung transplantation patients, thus limiting to some extent the generalizability of the results. Furthermore, though the data were prospectively collected, routine CXRs were obtained for all participants as in all prior studies, thereby leaving the question of 'real life' scenario applicability unanswered.

Although patients routinely underwent conscious sedation during FOB, using midazolam and fentanyl, the fact that all early-onset cases of pneumothorax requiring further intervention were symptomatic shows that premedication did not lead to symptom masking.

On multivariate analysis, in the current study chest pain aggravation and oxygen desaturation remained the only statistically significant predictors of the development of pneumothorax. Other factors evaluated, namely biopsy forceps diameter, lobe from which specimens were obtained, number of specimens taken, dyspnea and cough aggravation, and physician performing the FOB, had no predictive value. However, the small number of pneumothoraxes may well have masked an effect of one or more of these variables.

There are some limitations to the current study. First, in the current study, we used semiquantitative descriptors to measure patients' symptoms, as per definition the requirement to quantify a subjective parameter such as pain and dyspnea is problematic. By focusing on the change in each patient's symptom severity we were able to show a strong association between the change and the probability of suffering a pneumothorax. Furthermore, as the design of the current study was meant to optimally mimic a 'real-world' clinical scenario, the simplicity of the descriptive scale used (none, mild, moderate, and severe symptoms) is consistent with this aim and facilitates its applicability in daily practice. Second, following completion of patient recruitment, we have increasingly been utilizing propofol as the main sedative agent. The use of propofol during bronchoscopy is gaining favor worldwide with increasing evidence in the literature showing improved patient tolerance, shorter onset of action and recovery time, while maintaining similar efficacy and safety compared to midazolam [12]. Therefore, it would 
be expected that potential postprocedural symptom masking would actually be reduced with the use of propofol as opposed to midazolam. Another technology that was implemented in our institute after study completion and which is emerging in the practice of bronchoscopy is cryobiopsy sampling for the diagnosis of interstitial lung disease. The use of cryobiopsy sampling has been shown to increase the diagnostic yield but at the same time to significantly increase the risk of postprocedure pneumothorax, with some studies reporting an incidence rate of up to $23 \%$ [13]. Taking into consideration the high rate of procedure-related pneumothoraxes, patients undergoing cryobiopsy sampling should continue to have routine CXRs obtained until more data are available. Another concern is that, infrequently, patients might remain asymptomatic after FOB with TBB despite having a large pneumothorax and, therefore, could be at risk if CXR is not done. However, in the current study including 201 patients, all those having a pneumothorax requiring therapeutic intervention reported symptom aggravation following FOB with TBB. The only asymptomatic patient diagnosed with early-onset pneumothorax demonstrated a persistent oxygen desaturation of $>5 \%$. Of note, early CXR does not screen for late pneumothorax occurring $>2 \mathrm{~h}$ and up to a few days after FOB with TBB. Though a few cases of late pneumothorax exist in the literature [14], the 2 cases detected in this study were of negligible clinical significance. Furthermore, inherent to the definition of late pneumothorax, routine post-TBB CXR will not detect such an event since it is not present in the immediate post-FOB period. Last, pertaining to the potential value of using postprocedural chest ultrasonography for the detection of pneumothorax, this was clearly beyond the scope of our paper and we did not test this modality. It is important, however, to mention that the high accuracy of ultrasonography has been evaluated predominantly among acute trauma patients [15] and not in a population of patients with underlying lung pathologies undergoing procedures with a potential for iatrogenic pneumothorax. Indeed, in the largest study which specifically examined this issue [16], it was shown that as much as a quarter of the studies were of limited quality due to lung and pleural pathologies, necessitating the use of chest X-ray. Furthermore, as of today, ultrasound technology and the knowledge required to utilize it is still not routinely available in all the centers performing bronchoscopy worldwide.

A review of existing guidelines regarding the need for routine CXR after TBB reveals some inconsistencies with some advocating routine CXRs after every TBB procedure $[7,8]$ while others avoid any reference to the issue $[9,17]$. An exception to the rule are the latest guidelines on diagnostic flexible bronchoscopy publish by the British Thoracic Society (BTS) in 2013, which suggest that a CXR be obtained after TBB if the patient is symptomatic or if there is a clinical suspicion of possible pneumotho$\operatorname{rax}[8]$. This statement by the BTS is of the lowest recommendation rate and is based on nonanalytic studies and expert opinions.

It is our conclusion that routine CXR after TBB is not needed in asymptomatic patients who maintain a stable oxygenation level. This approach could decrease procedural cost as well as limit patient radiation exposure without any compromise in patient safety. We hope that the results of the present study will serve to strengthen the grade of the BTS guidelines recommendation and to stimulate a change of other existing guidelines regarding this commonly encountered clinical issue in pulmonary practice.

\section{Financial Disclosure and Conflicts of Interest}

All authors declare no conflicts of interest relevant to this publication. No financial support was received for this study.

References

1 Casal RF, Ost DE, Eapen GA: Flexible bronchoscopy. Clin Chest Med 2013;34:341-352.

$\checkmark 2$ Hanson RR, Zavala DC, Rhodes ML, Keim LW, Smith JD: Trans-bronchial biopsy via flexible fiberoptic bronchoscope; results in 164 patients. Am Rev Resp Dis 1974;114:6772.

- 3 Hernandez Blasco L, Sanchez Hernandez IM, Villena Garrido V, de Miguel Poch E, Nunez Delgado E, Alfaro Abreu J: Safety of the transbronchial biopsy in outpatients. Chest 1991; 99:562-565.

\footnotetext{
4 Izbicki G, Shitrit D, Yarmolovsky A, Bendayan D, Miller G, Fink G, Mazar A, Kramer MR: Is routine chest radiography after transbronchial biopsy necessary? Chest 2006;129:15611564.

5 Muzaffar A, Livingston DR, Golish JA, Mehta AC, Weidemann HP: The safety of outpatient transbronchial biopsy. Chest 1986;90:403405 .
} 
6 Sinha S, Guleria R, Pande JN, Pandey RM: Bronchoscopy in adults at a tertiary care center: indications and complications. J Indian Med Assoc 2004;102:152-154.

7 Febvre M, Trosini-Desert V, Atassi K, Hermant C, Colchen A, Raspaud C, Vergnon JM: Les bonnes pratiques de la bronchoscopie souple diagnostique, en 2007. Rev Mal Respir 2007;24:1363-1392.

8 Du Rand IA, Blaikley J, Booton R, Chaudhuri N, Gupta V, Khalid S, Mandal S, Martin J, Mills J, Navani N, Rahman NM, Wrightson JM, Munavvar M; British Thoracic Society Bronchoscopy Guideline Group: British Thoracic Society guidelines for diagnostic flexible bronchoscopy in adults. Thorax 2013;68:11i44.
9 Sokolowski JW, Burgher LW, Jones FL, Patterson JR, Selecky PA: Guidelines for fiberoptic bronchoscopy in adults. Am Rev Respir Dis 1987;136:1066.

10 Milam MG, Evins AE, Sahn SA: Immediate chest roentgenography following fiberoptic bronchoscopy. Chest 1989;96:477-479.

11 Frazier WD, Pope TL, Findley LJ: Pneumothorax following transbronchial biopsy. Chest 1990;97:539-540.

12 Jose RJ, Shaefi S, Navani N: Anesthesia for bronchoscopy. Curr Opin Anesthesiol 2014; 27:453-457.

13 Poletti V, Casoni GL, Gurioli C, Ryu JH, Tomassetti S: Lung cryobiopsy: a paradigm shift in diagnostic bronchoscopy? Respirology 2014;19:645-654.
4 Levy H, Kallenbach JM, Feldman C, Pincus P, Hurwitz M: Delayed pneumothorax after transbronchial lung biopsy. Thorax 1986;41: 647-648.

15 Alrajab S, Youssef AM, Akkus NI, Caldito G: Pleural ultrasonography versus chest radiology for diagnosis of pneumothorax: review of the literature and meta-analysis. Crit Care 2013;17:R208.

16 Shostak E, Brykla D, Krepp J, Pua B, Sanders A: Bedside ultrasonography in detection of post procedure pneumothorax. J Ultrasound Med 2013;32:1003-1009.

17 Ernst A, Silvestri GA, Johnstone D; ACCP Interventional Chest/Diagnostic Procedures Network Steering Committee: Interventional pulmonary procedures: guidelines from the American College of Chest Physicians. Chest 2003;123:1693-1717. 